J. Nonlinear Var. Anal. 3 (2019), No. 3, pp. 295-304

Available online at http://jnva.biemdas.com

https://doi.org/10.23952/jnva.3.2019.3.05

\title{
LARGE TIME BEHAVIOR OF SOLUTIONS TO WAVE EQUATIONS ARISING FROM THE LINEARIZED COMPRESSIBLE NAVIER-STOKES-POISSON SYSTEM
}

\author{
YANSHUO LI, JUN LIU, YU-ZHU WANG* \\ School of Mathematics and Statistics, \\ North China University of Water Resources and Electric Power, Zhengzhou 450011, China
}

\begin{abstract}
This paper is concerned the large time behavior of solutions to wave equations arising from the linearized compressible Navier-Stokes-Poisson (CNSP) system. By a detailed analysis of solution operators, we prove that global solutions could be approximated by the convolution of the heat kernel $\sin t$ together with the heat kernel $\cos t$.
\end{abstract}

Keywords. Linearized CNSP equations; Large time behavior; Heat kernel; Wave equation.

2010 Mathematics Subject Classification. 35L30, 35B40.

\section{INTRODUCTION}

Consider the following linear wave equation derived from linearized CNSP system

$$
\rho_{t t}-\Delta \rho-\Delta \rho_{t}+\rho=0
$$

with initial data

$$
\rho(0, x)=\rho_{0}(x), \quad \rho_{t}(0, x)=\rho_{1}(x),
$$

where $\rho=\rho(x, t)$ is the unknown function of $x \in \mathbb{R}^{n}(n \geq 1)$ and $t \geq 0$.

The CNSP system has the following form

$$
\left\{\begin{array}{l}
\rho_{t}+\operatorname{div}(\rho u)=0 \\
u_{t}+(u \cdot \nabla) u+\frac{\nabla P(\rho)}{\rho}+\nabla \Phi=\frac{\mu}{\rho} \Delta u+\frac{\mu+\lambda}{\rho} \nabla \operatorname{div} u \\
-\Delta \Phi=\rho-\bar{\rho},
\end{array}\right.
$$

where $x \in \mathbb{R}^{n}, t>0$. The unknown functions $\rho, u$ and $\Phi$ represent density, velocity and electrostatic potential, respectively. The viscosity coefficient $\mu$ and $\lambda$ satisfy $\mu>0$ and $2 \mu+\lambda>0$, and the background doping profile $\bar{\rho}$ is assumed to equal 1 . If $\rho=\rho-\bar{\rho}=\rho-1$, then (1.3) is transformed into

$$
\left\{\begin{array}{l}
\rho_{t}+\operatorname{div} u=f_{1}(u, \rho), \\
u_{t}-\mu \Delta u-(\mu+\lambda) \nabla \operatorname{div} u+\nabla \rho+\nabla(-\Delta)^{-1} \rho=f_{2}(u, \rho) .
\end{array}\right.
$$

* Corresponding author.

E-mail address: yuzhu108@163.com.

Received May 9, 2019; Accepted July 12, 2019.

(C)2019 Journal of Nonlinear and Variational Analysis 
Here, we only consider the linear part of (1.4)

$$
\left\{\begin{array}{l}
\rho_{t}+\operatorname{div} u=0 \\
u_{t}-\mu \Delta u-(\mu+\lambda) \nabla \operatorname{div} u+\nabla \rho+\nabla(-\Delta)^{-1} \rho=0
\end{array}\right.
$$

Performing div operator on $(1.5)_{2}$ and taking advantage of $(1.5)_{1}$ leads that

$$
\rho_{t t}-(2 \mu+\lambda) \Delta \rho_{t}-\Delta \rho+\rho=0 .
$$

Clearly, (1.1) follows provided that $2 \mu+\lambda=1$.

As the CNSP system has strong physical background, which attract mathematicians and physicians to study it and many results have been established, here we mainly pay attention to the results related to the asymptotic behavior. On one hand, some researchers focused on establishing the optimal time decay estimates of strong solutions to the CNSP system, see [2, 4, 10, 11, 12, 13, 14, 16, 19]. On the other hand, some researchers investigated the quasineutral limit and inviscid limit of CNSP system, see $[3,7,8,9,15]$.

Based on the spectral analysis of semigroups, Li, Matsumura and Zhang [10] showed that the density of CNSP system converges to its equilibrium state at the same $L^{2}$ and $L^{\infty}$ rate with the compressible Navier-Stokes system, and if the initial data are degenerate at the low frequency or decay fast at spatial infinity, the optimal convergence rates were also obtained by Li and Zhang [11]. Wang and Wang [16] established the global existence and new decay estimate of classical solution and gave the asymptotic behavior of corresponding linear problem in three and higher dimensions. Bie, Wang and Yao [2] established the optimal decay rate for the CNSP system in critical Besov spaces. Their results were improved by Shi and $\mathrm{Xu}$ in [14] later. If the doping profile are functions respect to space variable, Tan, Wang and Wang [12] proved the time decay rates for the solution provided that the initial perturbation in $L^{p}$ with $1 \leq p<\frac{3}{2}$. Feng and Liu [4] recently proved the stability of steady-state solutions based on the antisymmetric matrix techniques and induction argument.

For the general initial data, when the combined quasineutral and inviscid limit is considered, Wang and Jiang [15] proved the convergence of the global weak solution to the strong solution of incompressible Euler equations, and the concrete convergence rate was obtained by $\mathrm{Ju}, \mathrm{Li}$ and Wang [8]. In addition, the quasineutral limit of CNSP system with heat conductivity was also discussed by $\mathrm{Ju}, \mathrm{Li}$ and $\mathrm{Li}$ [9]. For the well-prepared data, Ju, Li and Wang [7] proved the global weak solution of CNSP system converges strongly to the strong solution of the incompressible Navier-Stokes equations and gave the convergence rate as well. We also mention that in the case of (1.3) with repulsion effect, that is, $\nabla \Phi$ with a negative coefficient, Bella [1] proved convergence of a weak solution to the stationary solution.

As we all know, an exact presentation of large time behavior of solutions to the CNSP system is still out of reach, we are interested in giving an exact asymptotic profile of solutions to problem (1.1)-(1.2). If $\rho$ in (1.1) is removed, (1.1) becomes dissipative wave equation, which has a long research history. By using the method developed in [5], Ikehata [6] obtained the asymptotic behavior of global solution could be expressed by convolution of fundamental solution of heat and free wave equation. Inspired by this work, we aim at constructing an asymptotic profile of solutions to problem (1.1)-(1.2), and the main results are stated as follows. 
Theorem 1.1. Assume that $\rho_{0}, \rho_{1} \in L^{1,1} \cap L^{2}$, and put

$$
E_{0}=\left\|\rho_{0}\right\|_{L^{1,1}}+\left\|\rho_{1}\right\|_{L^{1,1}}+\left\|\rho_{0}\right\|_{L^{2}}+\left\|\rho_{1}\right\|_{L^{2}} .
$$

Let $u$ be a solution to problem (1.1)-(1.2). Then for $t \gg 1$, there exists a constant $C_{0}$ depending on $E_{0}$ such that u satisfies the following

$$
\left\|\rho(t, x)-\left(R_{1} K(t, x) *(\delta(x) \sin t)+R_{0} K(t, x) *(\delta(x) \cos t)\right)\right\|_{L^{2}} \leq C_{0} t^{-\frac{n}{4}},
$$

where

$$
R_{j}=\int_{\mathbb{R}^{n}} \rho_{j}(x) d x(j=0,1), \quad K(t, x)=\mathscr{F}^{-1}\left[e^{-\frac{|\xi|^{2}}{2} t}\right] .
$$

Remark 1.1. Theorem 1.1 means that as $t \rightarrow \infty$, solutions to the problem (1.1)-(1.2) could be approximated by the convolution of the fundamental solutions to heat and the following equation

$$
\begin{cases}u_{t t}+u=0, & x \in \mathbb{R}^{n}, t>0, \\ u(0, x)=\delta(x), u_{t}(0, x)=\delta(x), & x \in \mathbb{R}^{n},\end{cases}
$$

where $\delta(x)$ is the usual dirac function.

Remark 1.2. The asymptotic profile (via the nonlinear term) of solutions to higher order wave equations has been considered in [17] and [18].

The rest part of this paper are unfolded as follows. Section 2 is devoted to make a detailed analysis of solution operators and discuss the decay estimates of solutions to the prolem (1.1)-(1.2). Finally, we prove Theorem 1.1 in Section 3.

In the last, we introduce some notations used in this paper. Let $\mathscr{F}[u]$ denote the Fourier transform of $u$ defined by

$$
\hat{u}(\xi)=\mathscr{F}[u](\xi):=\int_{\mathbb{R}^{n}} e^{-i \xi \cdot x} u(x) d x .
$$

We denote its inverse transform by $\mathscr{F}^{-1}$ and denote a generic positive constant by $C$ or $c$. For a nonnegative integer $k, \partial_{x}^{k}$ denotes the totality of all $k$ th-order derivatives with respect to $x \in \mathbb{R}^{n}$. For $1 \leq p \leq \infty$, let $L^{p}=L^{p}\left(\mathbb{R}^{n}\right)$ be the usual Lebesgue space with the norm $\|\cdot\|_{L^{p}}$. For $\gamma \in \mathbb{R}$, let $L^{1, \gamma}\left(\mathbb{R}^{n}\right)$ denote the weighted $L^{1}$ space with the norm

$$
\|f\|_{L^{1, \gamma}}=\int_{\mathbb{R}^{n}}(1+|x|)^{\gamma}|f(x)| d x .
$$

\section{ANALYSIS OF SOLUTION OPERATORS}

In this section, our aims are to make a detailed analysis of solution operators. First, we derive the mild solution to problem (1.1)-(1.2).

By taking the Fourier transform to (1.1)-(1.2), we have

$$
\begin{aligned}
& \hat{\rho}_{t t}+|\xi|^{2} \hat{\rho}_{t}+\left(|\xi|^{2}+1\right) \hat{\rho}=0, \\
& \hat{\rho}(0, \xi)=\hat{\rho}_{0}(\xi), \quad \hat{\rho}_{t}(0, \xi)=\hat{\rho}_{1}(\xi) .
\end{aligned}
$$

Obviously, the characteristic equation of (2.1) is as follows:

$$
\lambda^{2}+|\xi|^{2} \lambda+|\xi|^{2}+1=0
$$


Let $\lambda=\lambda_{ \pm}(\xi)$ be the corresponding eigenvalues. It is easy to see that

$$
\lambda_{ \pm}(\xi)=\frac{-|\xi|^{2} \pm \sqrt{|\xi|^{4}-4\left(|\xi|^{2}+1\right)}}{2} .
$$

Then the solution to problem (2.1)-(2.2) is expressed as

$$
\hat{\rho}(t, \xi)=\hat{G}(t, \xi) \hat{\rho}_{1}+\hat{H}(t, \xi) \hat{\rho}_{0},
$$

where

$$
\begin{aligned}
\hat{G}(t, \xi) & =\frac{1}{\lambda_{+}(\xi)-\lambda_{-}(\xi)}\left(e^{\lambda_{+}(\xi) t}-e^{\lambda_{-}(\xi) t}\right), \\
\hat{H}(t, \xi) & =\frac{1}{\lambda_{+}(\xi)-\lambda_{-}(\xi)}\left(\lambda_{+}(\xi) e^{\lambda_{-}(\xi) t}-\lambda_{-}(\xi) e^{\lambda_{+}(\xi) t}\right) .
\end{aligned}
$$

Taking $\mathscr{F}^{-1}$ to (2.4), we obtain the solution formula for problem (1.1)-(1.2)

$$
\rho(t)=G(t) * \rho_{1}(x)+H(t) * \rho_{0}(x) .
$$

In what follows, we would give a detailed analysis of solution operators by Fourier splitting frequency method. When $|\xi| \leq 1$, performing Taylor expansion on (2.5), we have

$$
\begin{gathered}
\hat{G}(t, \xi)=\frac{1}{v(\xi)} e^{-\frac{|\xi|^{2}}{2} t} \sin (v(\xi) t), \\
\hat{H}(t, \xi)=\frac{|\xi|^{2}}{2 v(\xi)} e^{-\frac{|\xi|^{2}}{2} t} \sin (v(\xi) t)+e^{-\frac{|\xi|^{2}}{2} t} \cos (v(\xi) t),
\end{gathered}
$$

where $v(\xi):=\sqrt{1+|\xi|^{2}-\frac{|\xi|^{4}}{4}}$. Thanks to the mean value theorem, we get

$$
\begin{aligned}
\frac{1}{v(\xi)} & =1-\frac{|\xi|^{2}-\frac{|\xi|^{4}}{4}}{2\left(1+\left(|\xi|^{2}-\frac{|\xi|^{4}}{4}\right) \theta_{1}\right)^{\frac{3}{2}}}, \\
\sin (v(\xi) t) & =\sin t+\cos \left(v(\xi) \theta_{2} t+\left(1-\theta_{2}\right) t\right)(v(\xi)-1) t
\end{aligned}
$$

where $\theta_{j}(j=1,2) \in(0,1)$. Inserting (2.9) into (2.7), we have

$$
\begin{aligned}
\hat{G}(t, \xi)= & e^{-\frac{|\xi|^{2}}{2} t} \sin t+e^{-\frac{|\xi|^{2}}{2} t} \cos \left(v(\xi) \theta_{2} t+\left(1-\theta_{2}\right) t\right)(v(\xi)-1) t \\
& -\frac{|\xi|^{2}}{2\left(1+\left(|\xi|^{2}-\frac{|\xi|^{4}}{4}\right) \theta_{1}\right)^{\frac{3}{2}}} e^{-\frac{|\xi|^{2}}{2} t} \sin t+\frac{|\xi|^{4}}{8\left(1+\left(|\xi|^{2}-\frac{|\xi|^{4}}{4}\right) \theta_{1}\right)^{\frac{3}{2}}} e^{-\frac{|\xi|^{2}}{2} t} \sin t \\
& -\frac{|\xi|^{2}}{2\left(1+\left(|\xi|^{2}-\frac{|\xi|^{4}}{4}\right) \theta_{1}\right)^{\frac{3}{2}}} e^{-\frac{|\xi|^{2}}{2} t} \cos \left(v(\xi) \theta_{2} t+\left(1-\theta_{2}\right) t\right)(v(\xi)-1) t \\
& +\frac{|\xi|^{4}}{8\left(1+\left(|\xi|^{2}-\frac{|\xi|^{4}}{4}\right) \theta_{1}\right)^{\frac{3}{2}}} e^{-\frac{|\xi|^{2}}{2} t} \cos \left(v(\xi) \theta_{2} t+\left(1-\theta_{2}\right) t\right)(v(\xi)-1) t \\
:= & e^{-\frac{|\xi|^{2}}{2} t \sin t+\sum_{i=1}^{5} I_{i} .}
\end{aligned}
$$

Similarly, resorting to the mean value theorem again, we readily obtain

$$
\cos (v(\xi) t)=\cos t-\sin \left(v(\xi) \theta_{3} t+\left(1-\theta_{3}\right) t\right)(v(\xi)-1) t
$$


where $\theta_{3} \in(0,1)$. Inserting (2.9) 1 and (2.11) into (2.8) yields

$$
\begin{aligned}
\hat{H}(t, \xi)= & e^{-\frac{|\xi|^{2}}{2} t} \cos t+|\xi|^{2} e^{-\frac{|\xi|^{2}}{2} t} \cos \left(v(\xi) \theta_{2} t+\left(1-\theta_{2}\right) t\right)(v(\xi)-1) t \\
& -\frac{|\xi|^{4}}{2\left(1+\left(|\xi|^{2}-\frac{|\xi|^{4}}{4}\right) \theta_{1}\right)^{\frac{3}{2}}} e^{-\frac{|\xi|^{2}}{2} t} \sin t+\frac{|\xi|^{6}}{8\left(1+\left(|\xi|^{2}-\frac{|\xi|^{4}}{4}\right) \theta_{1}\right)^{\frac{3}{2}}} e^{-\frac{|\xi|^{2}}{2} t} \sin t \\
& -\frac{|\xi|^{4}}{2\left(1+\left(|\xi|^{2}-\frac{|\xi|^{4}}{4}\right) \theta_{1}\right)^{\frac{3}{2}}} e^{-\frac{|\xi|^{2}}{2} t} \cos \left(v(\xi) \theta_{2} t+\left(1-\theta_{2}\right) t\right)(v(\xi)-1) t \\
& +\frac{|\xi|^{6}}{8\left(1+\left(|\xi|^{2}-\frac{|\xi|^{4}}{4}\right) \theta_{1}\right)^{\frac{3}{2}}} e^{-\frac{|\xi|^{2}}{2} t} \cos \left(v(\xi) \theta_{2} t+\left(1-\theta_{2}\right) t\right)(v(\xi)-1) t \\
& +|\xi|^{2} e^{-\frac{|\xi|^{2}}{2} t} \sin t+e^{-\frac{|\xi|^{2}}{2} t} \sin \left(v(\xi) \theta_{3} t+\left(1-\theta_{3}\right) t\right)(v(\xi)-1) t \\
:= & e^{-\frac{|\xi|^{2}}{2} t} \cos t+\sum_{i=6}^{12} I_{i} .
\end{aligned}
$$

It follows from the definition of the Fourier transform that

$$
\begin{aligned}
\hat{\rho}_{j}(t, \xi) & =\int_{\mathbb{R}^{n}} e^{-i x \cdot \xi t} \rho_{j}(x) d x \\
& =\int_{\mathbb{R}^{n}}(\cos (x \cdot \xi)-1) \rho_{j}(x) d x-i \int_{\mathbb{R}^{n}} \sin (x \cdot \xi) \rho_{j}(x) d x+\int_{\mathbb{R}^{n}} \rho_{j}(x) d x \\
& =P_{j}(\xi)-i Q_{j}(\xi)+R_{j} \quad(j=0,1) .
\end{aligned}
$$

By (2.4), we have

$$
\begin{aligned}
& \hat{\rho}(t, \xi)-e^{-\frac{|\xi|^{2}}{2} t}\left(R_{1} \sin t+R_{0} \cos t\right) \\
& =e^{-\frac{|\xi|^{2}}{2} t} \sin t\left(P_{1}(\xi)-i Q_{1}(\xi)\right)+e^{-\frac{|\xi|^{2}}{2} t} \cos t\left(P_{0}(\xi)-i Q_{0}(\xi)\right) \\
& \quad+\sum_{i=1}^{5} I_{i}\left(P_{1}(\xi)-i Q_{1}(\xi)\right)+\sum_{i=6}^{12} I_{i}\left(P_{0}(\xi)-i Q_{0}(\xi)\right) \\
& \quad+\sum_{i=1}^{5} I_{i} R_{1}+\sum_{i=6}^{12} I_{i} R_{0} .
\end{aligned}
$$

Before proving Theorem 1.1, we pause here to discuss the decay estimate of solutions to problem (1.1)-(1.2). First, we apply the Fourier expansion to (2.5) and obtain

$$
\begin{array}{ll}
|G(t)| \leq C e^{-c|\xi|^{2} t}, & |H(t)| \leq C e^{-c|\xi|^{2} t} \\
\left|G_{t}(t)\right| \leq C e^{-c|\xi|^{2} t}, & \left|H_{t}(t)\right| \leq C e^{-c|\xi|^{2} t}
\end{array}
$$

for $|\xi| \leq 1$ and

$$
\begin{array}{ll}
|G(t)| \leq C|\xi|^{-2} e^{-c t}, & |H(t)| \leq C e^{-c t} \\
\left|G_{t}(t)\right| \leq C e^{-c t}, & \left|H_{t}(t)\right| \leq C|\xi|^{2} e^{-c t}
\end{array}
$$

for $|\xi| \geq 1$

Then we introduce the following lemma established in [5].

Lemma 2.1. Let $\gamma \in[0,1]$. Assume that $u \in L^{1, \gamma}$, there holds that

$$
|\hat{u}(\xi)| \leq C_{\gamma}|\xi|^{\gamma}\|u\|_{L^{1, \gamma}}+\left|\int_{\mathbb{R}^{n}} u(x) d x\right| .
$$


The solutions to problem (1.1)-(1.2) satisfy the following decay estimates.

Theorem 2.1. Assume that $\rho_{0} \in L^{1,1} \cap H^{2}, \rho_{1} \in L^{1,1} \cap L^{2}$. Let $\rho$ be the solution to (1.1)-(1.2). Then we have

and

$$
\begin{aligned}
\|\rho(t)\|_{L^{2}} \leq & C\left(\left\|\rho_{0}\right\|_{L^{1,1}}+\left\|\rho_{1}\right\|_{L^{1,1}}\right)(1+t)^{-\frac{n}{4}-\frac{1}{2}}+C e^{-c t}\left(\left\|\rho_{0}\right\|_{L^{2}}+\left\|\rho_{1}\right\|_{L^{2}}\right) \\
& +C(1+t)^{-\frac{n}{4}}\left(\left|\int_{\mathbb{R}^{n}} \rho_{0}(x) d x\right|+\left|\int_{\mathbb{R}^{n}} \rho_{1}(x) d x\right|\right)
\end{aligned}
$$

$$
\begin{aligned}
\left\|\rho_{t}(t)\right\|_{L^{2}} \leq & C\left(\left\|\rho_{0}\right\|_{L^{1,1}}+\left\|\rho_{1}\right\|_{L^{1,1}}\right)(1+t)^{-\frac{n}{4}-\frac{1}{2}}+C e^{-c t}\left(\left\|\rho_{0}\right\|_{H^{2}}+\left\|\rho_{1}\right\|_{L^{2}}\right) \\
& +C(1+t)^{-\frac{n}{4}}\left(\left|\int_{\mathbb{R}^{n}} \rho_{0}(x) d x\right|+\left|\int_{\mathbb{R}^{n}} \rho_{1}(x) d x\right|\right)
\end{aligned}
$$

To prove Theorem 2.1, it suffices to prove the following lemma.

Lemma 2.2. Let $k \geq 0$ be integer and $\gamma \in[0,1]$. Then

$$
\begin{aligned}
& \left\|G(t) * \rho_{1}\right\|_{L^{2}} \leq C\left\|\rho_{1}\right\|_{L^{1, \gamma}}(1+t)^{-\frac{n}{4}-\frac{\gamma}{2}}+C e^{-c t}\left\|\rho_{1}\right\|_{L^{2}}+C(1+t)^{-\frac{n}{4}}\left|\int_{\mathbb{R}^{n}} \rho_{1} d x\right|, \\
& \left\|H(t) * \rho_{0}\right\|_{L^{2}} \leq C\left\|\rho_{0}\right\|_{L^{1, \gamma}}(1+t)^{-\frac{n}{4}-\frac{\gamma}{2}}+C e^{-c t}\left\|\rho_{0}\right\|_{L^{2}}+C(1+t)^{-\frac{n}{4}}\left|\int_{\mathbb{R}^{n}} \rho_{0} d x\right|, \\
& \left\|G_{t}(t) * \rho_{1}\right\|_{L^{2}} \leq C\left\|\rho_{1}\right\|_{L^{1, \gamma}}(1+t)^{-\frac{n}{4}-\frac{\gamma}{2}}+C e^{-c t}\left\|\rho_{1}\right\|_{L^{2}}+C(1+t)^{-\frac{n}{4}}\left|\int_{\mathbb{R}^{n}} \rho_{1} d x\right|, \\
& \left\|H_{t}(t) * \rho_{0}\right\|_{L^{2}} \leq C\left\|\rho_{0}\right\|_{L^{1, \gamma}}(1+t)^{-\frac{n}{4}-\frac{\gamma}{2}}+C e^{-c t}\left\|\partial_{x}^{2} \rho_{0}\right\|_{L^{2}}+C(1+t)^{-\frac{n}{4}}\left|\int_{\mathbb{R}^{n}} \rho_{0} d x\right| .
\end{aligned}
$$

Proof. We only prove (2.17). Thanks to (2.15), (2.16) and Plancherel theorem, we have

$$
\begin{aligned}
\left\|G(t) * \rho_{1}\right\|_{L^{2}}^{2} & =\int_{|\xi| \leq 1}|\hat{G}(\xi, t)|^{2}\left|\hat{\rho}_{1}(\xi)\right|^{2} d \xi+\int_{|\xi| \geq 1}|\hat{G}(\xi, t)|^{2}\left|\hat{\rho}_{1}(\xi)\right|^{2} d \xi \\
& \leq C \int_{|\xi| \leq 1} e^{-c|\xi|^{2} t}\left|\hat{\rho}_{1}(\xi)\right|^{2} d \xi+C e^{-c t} \int_{|\xi| \geq 1}|\xi|^{-4}\left|\hat{\rho}_{1}(\xi)\right|^{2} d \xi \\
& \leq C\left\|\rho_{1}\right\|_{L^{1, \gamma}}^{2} \int_{|\xi| \leq 1}|\xi|^{2 \gamma} e^{-c|\xi|^{2} t} d \xi+C\left|\int_{\mathbb{R}^{d}} \rho_{1} d x\right|^{2} \int_{|\xi| \leq 1} e^{-c|\xi|^{2} t} d \xi+C e^{-c t}\left\|\rho_{1}\right\|_{L^{2}}^{2} \\
& \leq C\left\|\rho_{1}\right\|_{L^{1, \gamma}}^{2}(1+t)^{-\frac{n}{4}-\frac{\gamma}{2}}+C\left|\int_{\mathbb{R}^{d}} \rho_{1} d x\right|^{2}(1+t)^{-\frac{n}{4}}+C e^{-c t}\left\|\rho_{1}\right\|_{L^{2}}^{2} .
\end{aligned}
$$

Then (2.17) clearly follows. Thus Lemma 2.2 is proved.

\section{Proof of Theorem 1.1}

In order to prove Theorem 1.1, we need the following lemma has been established in [6].

Lemma 3.1. For any $\xi \in \mathbb{R}^{n}$ and $j=0,1$, the following holds

$$
\begin{aligned}
& \left|P_{j}(\xi)\right| \leq \Lambda_{1}|\xi|\left\|u_{j}\right\|_{L^{1,1}}, \\
& \left|Q_{j}(\xi)\right| \leq \Lambda_{2}|\xi||| u_{j} \|_{L^{1,1}}
\end{aligned}
$$

where

$$
\Lambda_{1}:=\sup _{\tau \neq 0} \frac{|1-\cos \tau|}{|\tau|}<+\infty, \quad \Lambda_{2}:=\sup _{\tau \neq 0} \frac{|\sin \tau|}{|\tau|}<+\infty .
$$


In what follows, we would like to give a detailed proof of Theorem 1.1.

Proof. Owing to Plancherel theorem, we have

$$
\begin{aligned}
& \int_{\mathbb{R}^{n}} \mid \rho(t, \cdot)-\left(R_{1} K(t, x) *(\delta(x) \sin t)+\left.R_{0} K(t, x) *(\delta(x) \cos t)\right|^{2} d x\right. \\
& =\int_{|\xi| \leq 1}\left|\hat{\rho}(t, \cdot)-e^{-\frac{|\xi|^{2}}{2} t}\left(R_{1} \sin t+R_{0} \cos t\right)\right|^{2} d \xi \\
& \quad+\int_{|\xi| \geq 1}\left|\hat{\rho}(t, \cdot)-e^{-\frac{|\xi|^{2}}{2} t}\left(R_{1} \sin t+R_{0} \cos t\right)\right|^{2} d \xi \\
& :=J_{1}+J_{2} .
\end{aligned}
$$

It follows from (2.14) that

$$
\begin{aligned}
J_{1} \leq & \int_{|\xi| \leq 1} e^{-|\xi|^{2} t}|\sin t|^{2}\left|P_{1}(\xi)-i Q_{1}(\xi)\right|^{2} d \xi \\
& +\int_{|\xi| \leq 1} e^{-|\xi|^{2} t}|\cos t|^{2}\left|P_{0}(\xi)-i Q_{0}(\xi)\right|^{2} d \xi \\
& +\sum_{i=1}^{5} \int_{|\xi| \leq 1}\left|I_{i} R_{1}\right|^{2} d \xi+\sum_{i=1}^{5} \int_{|\xi| \leq 1}\left|I_{i}\left(P_{1}(\xi)-i Q_{1}(\xi)\right)\right|^{2} d \xi \\
& +\sum_{i=6}^{12} \int_{|\xi| \leq 1}\left|I_{i} R_{0}\right|^{2} d \xi+\sum_{i=6}^{12} \int_{|\xi| \leq 1}\left|I_{i}\left(P_{0}(\xi)-i Q_{0}(\xi)\right)\right|^{2} d \xi
\end{aligned}
$$

Thanks to Lemma 3.1, we have

$$
\begin{aligned}
\int_{|\xi| \leq 1} e^{-|\xi|^{2} t}|\sin t|^{2}\left|P_{1}(\xi)-i Q_{1}(\xi)\right|^{2} d \xi & \leq C \int_{|\xi| \leq 1} e^{-|\xi|^{2} t}\left(\left|P_{1}(\xi)\right|^{2}+\left|Q_{1}(\xi)\right|^{2}\right) d \xi \\
& \leq C\left(\Lambda_{1}^{2}+\Lambda_{2}^{2}\right)\left\|\rho_{1}\right\|_{L^{1,1}}^{2} \int_{|\xi| \leq 1}|\xi|^{2} e^{-|\xi|^{2} t} d \xi \\
& \leq C\left\|\rho_{1}\right\|_{L^{1,1}}^{2}(1+t)^{-\frac{n}{2}-1}
\end{aligned}
$$

and

$$
\begin{aligned}
\int_{|\xi| \leq 1} e^{-|\xi|^{2} t}|\cos t|^{2}\left|P_{0}(\xi)-i Q_{0}(\xi)\right|^{2} d \xi & \leq C \int_{|\xi| \leq 1} e^{-|\xi|^{2} t}\left(\left|P_{0}(\xi)\right|^{2}+\left|Q_{0}(\xi)\right|^{2}\right) d \xi \\
& \leq C\left(\Lambda_{1}^{2}+\Lambda_{2}^{2}\right)\left\|\rho_{0}\right\|_{L^{1,1}}^{2} \int_{|\xi| \leq 1}|\xi|^{2} e^{-|\xi|^{2} t} d \xi \\
& \leq C\left\|\rho_{0}\right\|_{L^{1,1}}^{2}(1+t)^{-\frac{n}{2}-1} .
\end{aligned}
$$

Since $v(\xi)-1=O\left(|\xi|^{2}\right)$ when $|\xi| \leq 1$, we have

$$
\begin{aligned}
\int_{|\xi| \leq 1}\left|I_{1} R_{1}\right|^{2} d \xi & \leq C\left|R_{1}\right|^{2} t^{2} \int_{|\xi| \leq 1}|\xi|^{4} e^{-|\xi|^{2} t} d \xi \\
& \leq C\left|R_{1}\right|^{2} t^{-\frac{n}{2}} \\
\int_{|\xi| \leq 1}\left|I_{2} R_{1}\right|^{2} d \xi & \leq C\left|R_{1}\right|^{2} \int_{|\xi| \leq 1}|\xi|^{4} e^{-|\xi|^{2} t} d \xi \\
& \leq C\left|R_{1}\right|^{2} t^{-\frac{n}{2}-2}, \\
\int_{|\xi| \leq 1}\left|I_{3} R_{1}\right|^{2} d \xi & \leq C\left|R_{1}\right|^{2} \int_{|\xi| \leq 1}|\xi|^{8} e^{-|\xi|^{2} t} d \xi \\
& \leq C\left|R_{1}\right|^{2} t^{-\frac{n}{2}-4},
\end{aligned}
$$


and

$$
\begin{aligned}
\int_{|\xi| \leq 1}\left|I_{4} R_{1}\right|^{2} d \xi & \leq C\left|R_{1}\right|^{2} t^{2} \int_{|\xi| \leq 1}|\xi|^{8} e^{-|\xi|^{2} t} d \xi \\
& \leq C\left|R_{1}\right|^{2} t^{-\frac{n}{2}-2}
\end{aligned}
$$

$$
\begin{aligned}
\int_{|\xi| \leq 1}\left|I_{5} R_{1}\right|^{2} d \xi & \leq C\left|R_{1}\right|^{2} t^{2} \int_{|\xi| \leq 1}|\xi|^{12} e^{-|\xi|^{2} t} d \xi \\
& \leq C\left|R_{1}\right|^{2} t^{-\frac{n}{2}-4} .
\end{aligned}
$$

Resorting to Lemma 3.1 again, we have

$$
\begin{aligned}
\int_{|\xi| \leq 1}\left|I_{1}\left(P_{1}(\xi)-i Q_{1}(\xi)\right)\right|^{2} d \xi & \leq C\left(\Lambda_{1}^{2}+\Lambda_{2}^{2}\right)\left\|\rho_{1}\right\|_{L^{1,1}}^{2} t^{2} \int_{|\xi| \leq 1}|\xi|^{6} e^{-|\xi|^{2} t} d \xi \\
& \leq C\left\|\rho_{1}\right\|_{L^{1,1}}^{2} t^{-\frac{n}{2}-1}, \\
\int_{|\xi| \leq 1}\left|I_{2}\left(P_{1}(\xi)-i Q_{1}(\xi)\right)\right|^{2} d \xi & \leq C\left(\Lambda_{1}^{2}+\Lambda_{2}^{2}\right)\left\|u_{1}\right\|_{L^{1,1}}^{2} \int_{|\xi| \leq 1}|\xi|^{6} e^{-|\xi|^{2} t} d \xi \\
& \leq C\left\|\rho_{1}\right\|_{L^{1,1}}^{2} t^{-\frac{n}{2}-3}, \\
\int_{|\xi| \leq 1}\left|I_{3}\left(P_{1}(\xi)-i Q_{1}(\xi)\right)\right|^{2} d \xi & \leq C\left(\Lambda_{1}^{2}+\Lambda_{2}^{2}\right)\left\|u_{1}\right\|_{L^{1,1}}^{2} \int_{|\xi| \leq 1}|\xi|^{10} e^{-|\xi|^{2} t} d \xi \\
& \leq C\left\|\rho_{1}\right\|_{L^{1,1}}^{2} t^{-\frac{n}{2}-5}, \\
\int_{|\xi| \leq 1}\left|I_{4}\left(P_{1}(\xi)-i Q_{1}(\xi)\right)\right|^{2} d \xi & \leq C\left(\Lambda_{1}^{2}+\Lambda_{2}^{2}\right)\left\|\rho_{1}\right\|_{L^{1,1}}^{2} t^{2} \int_{|\xi| \leq 1}|\xi|^{10} e^{-|\xi|^{2} t} d \xi \\
& \leq C\left\|\rho_{1}\right\|_{L^{1,1}}^{2} t^{-\frac{n}{2}-3}
\end{aligned}
$$

and

$$
\begin{aligned}
\int_{|\xi| \leq 1}\left|I_{5}\left(P_{1}(\xi)-i Q_{1}(\xi)\right)\right|^{2} d \xi & \leq C\left(\Lambda_{1}^{2}+\Lambda_{2}^{2}\right)\left\|\rho_{1}\right\|_{L^{1,1}}^{2} t^{2} \int_{|\xi| \leq 1}|\xi|^{14} e^{-|\xi|^{2} t} d \xi \\
& \leq C\left\|\rho_{1}\right\|_{L^{1,1}}^{2} t^{-\frac{n}{2}-5} .
\end{aligned}
$$

Similarly, we can get

$$
\begin{aligned}
\int_{|\xi| \leq 1}\left|I_{6} R_{0}\right|^{2} d \xi & \leq C\left|R_{0}\right|^{2} t^{2} \int_{|\xi| \leq 1}|\xi|^{8} e^{-|\xi|^{2} t} d \xi \\
& \leq C\left|R_{0}\right|^{2} t^{-\frac{n}{2}-2}, \\
\int_{|\xi| \leq 1}\left|I_{7} R_{0}\right|^{2} d \xi & \leq C\left|R_{0}\right|^{2} \int_{|\xi| \leq 1}|\xi|^{8} e^{-|\xi|^{2} t} d \xi \\
& \leq C\left|R_{0}\right|^{2} t^{-\frac{n}{2}-4}, \\
\int_{|\xi| \leq 1}\left|I_{8} R_{0}\right|^{2} d \xi & \leq C\left|R_{0}\right|^{2} \int_{|\xi| \leq 1}|\xi|^{12} e^{-|\xi|^{2} t} d \xi \\
& \leq C\left|R_{0}\right|^{2} t^{-\frac{n}{2}-6}, \\
\int_{|\xi| \leq 1}\left|I_{9} R_{0}\right|^{2} d \xi & \leq C\left|R_{0}\right|^{2} t^{2} \int_{|\xi| \leq 1}|\xi|^{12} e^{-|\xi|^{2} t} d \xi \\
& \leq C\left|R_{0}\right|^{2} t^{-\frac{n}{2}-4}, \\
\int_{|\xi| \leq 1}\left|I_{10} R_{0}\right|^{2} d \xi & \leq C\left|R_{0}\right|^{2} t^{2} \int_{|\xi| \leq 1}|\xi|^{16} e^{-|\xi|^{2} t} d \xi \\
& \leq C\left|R_{0}\right|^{2} t^{-\frac{n}{2}-6},
\end{aligned}
$$




$$
\begin{aligned}
& \int_{|\xi| \leq 1}\left|I_{11} R_{0}\right|^{2} d \xi \leq C\left|R_{0}\right|^{2} \int_{|\xi| \leq 1}|\xi|^{4} e^{-|\xi|^{2} t} d \xi \\
& \leq C\left|R_{0}\right|^{2} t^{-\frac{n}{2}-2} \\
& \int_{|\xi| \leq 1}\left|I_{12} R_{0}\right|^{2} d \xi \leq C\left|R_{0}\right|^{2} t^{2} \int_{|\xi| \leq 1}|\xi|^{4} e^{-|\xi|^{2} t} d \xi \\
& \leq C\left|R_{0}\right|^{2} t^{-\frac{n}{2}}, \\
& \int_{|\xi| \leq 1}\left|I_{6}\left(P_{0}(\xi)-i Q_{0}(\xi)\right)\right|^{2} d \xi \leq C\left(\Lambda_{1}^{2}+\Lambda_{2}^{2}\right)\left\|u_{0}\right\|_{L^{1,1}}^{2} t^{2} \int_{|\xi| \leq 1}|\xi|^{10} e^{-|\xi|^{2} t} d \xi \\
& \leq C\left\|\rho_{0}\right\|_{L^{1,1}}^{2} t^{-\frac{n}{2}-3}, \\
& \int_{|\xi| \leq 1}\left|I_{7}\left(P_{0}(\xi)-i Q_{0}(\xi)\right)\right|^{2} d \xi \leq C\left(\Lambda_{1}^{2}+\Lambda_{2}^{2}\right)\left\|\rho_{0}\right\|_{L^{1,1}}^{2} \int_{|\xi| \leq 1}|\xi|^{10} e^{-|\xi|^{2} t} d \xi \\
& \leq C\left\|\rho_{0}\right\|_{L^{1,1}}^{2} t^{-\frac{n}{2}-5}, \\
& \int_{|\xi| \leq 1}\left|I_{8}\left(P_{0}(\xi)-i Q_{0}(\xi)\right)\right|^{2} d \xi \leq C\left(\Lambda_{1}^{2}+\Lambda_{2}^{2}\right)\left\|\rho_{0}\right\|_{L^{1,1}}^{2} \int_{|\xi| \leq 1}|\xi|^{14} e^{-|\xi|^{2} t} d \xi \\
& \leq C\left\|\rho_{0}\right\|_{L^{1,1}}^{2} t^{-\frac{n}{2}-7}, \\
& \int_{|\xi| \leq 1}\left|I_{9}\left(P_{0}(\xi)-i Q_{0}(\xi)\right)\right|^{2} d \xi \leq C\left(\Lambda_{1}^{2}+\Lambda_{2}^{2}\right)\left\|\rho_{0}\right\|_{L^{1,1}}^{2} t^{2} \int_{|\xi| \leq 1}|\xi|^{14} e^{-|\xi|^{2} t} d \xi \\
& \leq C\left\|\rho_{0}\right\|_{L^{1,1}}^{2} t^{-\frac{n}{2}-5}, \\
& \int_{|\xi| \leq 1}\left|I_{10}\left(P_{0}(\xi)-i Q_{0}(\xi)\right)\right|^{2} d \xi \leq C\left(\Lambda_{1}^{2}+\Lambda_{2}^{2}\right)\left\|\rho_{0}\right\|_{L^{1,1}}^{2} t^{2} \int_{|\xi| \leq 1}|\xi|^{18} e^{-|\xi|^{2} t} d \xi \\
& \leq C\left\|\rho_{0}\right\|_{L^{1,1}}^{2} t^{-\frac{n}{2}-7}, \\
& \int_{|\xi| \leq 1}\left|I_{11}\left(P_{0}(\xi)-i Q_{0}(\xi)\right)\right|^{2} d \xi \leq C\left(\Lambda_{1}^{2}+\Lambda_{2}^{2}\right)\left\|\rho_{0}\right\|_{L^{1,1}}^{2} \int_{|\xi| \leq 1}|\xi|^{6} e^{-|\xi|^{2} t} d \xi \\
& \leq C\left\|\rho_{0}\right\|_{L^{1,1}}^{2} t^{-\frac{n}{2}-3}
\end{aligned}
$$

and

$$
\begin{aligned}
\int_{|\xi| \leq 1}\left|I_{12}\left(P_{0}(\xi)-i Q_{0}(\xi)\right)\right|^{2} d \xi & \leq C\left(\Lambda_{1}^{2}+\Lambda_{2}^{2}\right)\left\|\rho_{0}\right\|_{L^{1,1}}^{2} t^{2} \int_{|\xi| \leq 1}|\xi|^{6} e^{-|\xi|^{2} t} d \xi \\
& \leq C\left\|\rho_{0}\right\|_{L^{1,1}}^{2} t^{-\frac{n}{2}-1} .
\end{aligned}
$$

Substituting above estimates into (3.2) gives

$$
J_{1} \leq C t^{-\frac{n}{2}}\left(\left\|\rho_{0}\right\|_{L^{1,1}}^{2}+\left\|\rho_{1}\right\|_{L^{1,1}}^{2}\right) .
$$

Now, it remains to estimate $J_{2}$. By (2.4) and (2.16), we arrive at

$$
\begin{aligned}
J_{2} & \leq \int_{|\xi| \geq 1}|\hat{\rho}(\xi, t)|^{2} d \xi+\left|R_{1}\right|^{2} \int_{|\xi| \geq 1} e^{-|\xi|^{2} t}|\sin t|^{2} d \xi+\left|R_{0}\right|^{2} \int_{|\xi| \geq 1} e^{-|\xi|^{2} t}|\cos t|^{2} d \xi \\
& \leq C e^{-c t} \int_{|\xi| \geq 1}\left(\frac{\left|\hat{\rho}_{1}\right|^{2}}{|\xi|^{4}}+\left|\hat{\rho}_{0}\right|^{2}\right) d \xi+C\left(\left|R_{1}\right|^{2}+\left|R_{0}\right|^{2}\right) \int_{|\xi| \geq 1} e^{-|\xi|^{2} t} d \xi \\
& \leq C e^{-c t}\left(\left\|\rho_{1}\right\|_{2}^{2}+\left\|\rho_{0}\right\|_{2}^{2}\right)+C\left(\left|R_{1}\right|^{2}+\left|R_{0}\right|^{2}\right) e^{-\frac{1}{2} t} t^{-\frac{n}{2}} \\
& \leq C e^{-c t}\left(\left\|\rho_{1}\right\|_{2}^{2}+\left\|\rho_{0}\right\|_{2}^{2}+\left\|\rho_{1}\right\|_{L^{1,1}}^{2}+\left\|\rho_{0}\right\|_{L^{1,1}}^{2}\right) .
\end{aligned}
$$

Substituting (3.3) and (3.4) into (3.1), we readily obtain (1.6). Therefore, the whole proof of Theorem 1.1 is completed. 


\section{Acknowledgments}

This work was supported in part by the Innovative Subject of Graduate of North China University of Water Resources and Electric Power (Grant No. YK2018-28).

\section{REFERENCES}

[1] P. Bella, Long time behavior of weak solutions to Navier-Stokes-Poisson system, J. Math. Fluid Mech. 14 (2012), $279-294$.

[2] Q. Bie, Q. Wang, Z.-A. Yao, Optimal decay rate for the compressible Navier-Stokes-Poisson system in the critical framework, J. Differential Equations 263 (2017) 8391-8417.

[3] D. Donatelli, P. Marcati, A quasineutral type limit for the Navier-Stokes-Poisson system with large data, Nonliearity 21 (2007), 135-148.

[4] Y.H. Feng, C. M. Liu, Stability of steady-state solutions to Navier-Stokes-Poisson systems, J. Math. Anal. Appl. 462 (2018), 1679-1694.

[5] R. Ikehata, New decay estimates for linear damped wave equations and its application to nonlinear prolblem, Math. Methods Appl. Sci. 27 (2004), 865-889.

[6] R. Ikehata, Asymptotic profiles for wave equations with strong damping, J. Differential Equations 257 (2014), $2159-2177$.

[7] Q.C. Ju, F.C. Li, S. Wang, Convergence of the Navier-Stokes-Poisson system to the incompressible Navier-Stokes equations, J. Math. Phys. 49 (2008), 073515.

[8] Q.C. Ju, Y. Li, S. Wang, Rate of convergence from the Navier-Stokes-Poisson system to the incompressible Euler equations, J. Math. Phys. 50 (2009), 013533.

[9] Q.C. Ju, F.C. Li, H.L. Li, The quasineutral limit of compressible Navier-Stokes-Poisson system with heat conductivity and general initial data, J. Differential Equations 247 (2009), 203-224.

[10] H.L. Li, A. Matsumura, G.J. Zhang, Optimal decay rate of the compressible Navier-Stokes-Poisson system in $\mathbb{R}^{3}$, Arch. Ration. Mech. Anal. 196 (2010), 681-713.

[11] H.L. Li, T. Zhang, Large time behavior of solutions to 3D compressible Navier-Stokes-Poisson system, Sci. China Math. 55 (2012), 159-177.

[12] Z. Tan, Y.J. Wang, Y. Wang, Stability of steady states of the Navier-Stokes-Poisson equations with non-flat doping profile, SIAM. J. Math. Anal. 47 (2015), 179-209.

[13] Z. Tan, Y. Wang, X. Zhang, Large time behavior of solutions to the non-isentropic compressible Navier-Stokes-Poisson system in $\mathbb{R}^{3}$, Kinet. Relat. Models 5 (2012), 615-638.

[14] W. Shi, J. Xu, A sharp time-weighted inequality for the compressible Navier-Stokes-Poisson system in the critical $L^{p}$ framework, J. Differential Equations 266 (2019), 6426-6458.

[15] S. Wang, S. Jiang, The convergence of the Navier-Stokes-Poisson system to the incompressible Euler equations, Commun. Partial Differ. Equ. 31 (2006), 571-591.

[16] Y.-Z. Wang, K.Y. Wang, Asymptotic behavior of classical solutions to the compressible Navier-Stokes-Poisson equations in three and higher dimensions, J. Differential Equations 259 (2015), 25-47.

[17] Y.-Z. Wang, S. Chen, Asymptotic profile of solutions to the double dispersion equation, Nonlinear Anal. 134 (2016), 236-254.

[18] Y.-Z. Wang, C.H. Wei, Asymptotic profile of global solutions to the generalized double dispersion equation via the nonlinear term, Z. Angew. Math. Phys. 69 (2018), 1-17.

[19] L. Xiao, H.L. Li, Compressible Navier-Stokes-Poisson system equations, Acta. Math. Sci. 30B (2010), 1937-1948. 